\title{
Analyse ergonomique de l'activité de conduite moto lors de la pratique de l'inter-files en région parisienne
}

Ergonomic study of riding a motorcycle between traffic lanes: the Parisian example

\section{Samuel Aupetit et Stéphane Espié}

\section{OpenEdition}

Journals

Édition électronique

URL : http://journals.openedition.org/activites/322

DOI : 10.4000/activites.322

ISSN : $1765-2723$

Éditeur

ARPACT - Association Recherches et Pratiques sur les ACTivités

\section{Référence électronique}

Samuel Aupetit et Stéphane Espié, « Analyse ergonomique de l'activité de conduite moto lors de la pratique de l'inter-files en région parisienne », Activités [En ligne], 9-2 I Octobre 2012, mis en ligne le 15 octobre 2012, consulté le 30 avril 2019. URL : http://journals.openedition.org/activites/322 ; DOI : 10.4000 /activites.322

\section{(c) $\oplus \Theta \Theta$}

Activités est mis à disposition selon les termes de la licence Creative Commons Attribution - Pas d'Utilisation Commerciale - Pas de Modification 4.0 International. 


\title{
Analyse ergonomique de l'activité de conduite moto lors de la pratique de l'inter-files en région parisienne
}

\author{
Samuel Aupetit
}

Université Paris-Est, IFSTTAR, IM, LEPSIS, F-75732 Paris - $\underline{\text { samuel.aupetit@ifsttar.fr }}$

Stéphane Espié

Université Paris-Est, IFSTTAR, IM, F-75732 Paris - stephane.espie@ifsttar.fr

\begin{abstract}
Ergonomic study of riding a motorcycle between traffic lanes: the Parisian example. This paper presents a naturalistic study of how motorcyclists behave whilst commuting in the Paris region. The study focuses on lane-splitting which consists in riding between traffic lanes. This phenomenon has never been subjected to any detailed scientific analysis. In an ergonomic study, for one month 11 motorcyclists drove a motorbike equipped with cameras. The video recordings and the conduct of self-confrontation interviews enabled the description of the driving contexts. The results concern the description of the motorcyclists' activity, the importance of lane-splitting in their daily journeys, and the study of the organizing elements of their behaviors. These results are discussed in terms of a better understanding of lane-splitting and in terms of the improvement of the existing situations: the data question the possibility of a law regarding this practice and demonstrate the utility of training.
\end{abstract}

\section{KEYWORDS}

motorcycling, lane-splitting, self-confrontation interview, ergonomics, road safety

\section{1.- Introduction}

Les risques liés à la conduite d'un deux-roues à moteur (2RM) constituent un enjeu majeur de santé publique en France. En région parisienne, les conducteurs de 2RM sont impliqués dans $50 \%$ des accidents de la route et représentent $40 \%$ des victimes en 2009 (Observatoire National Interministériel de la Sécurité Routière ONISR, 2010). L'augmentation du nombre de conducteurs de 2RM dans les agglomérations est appelée à se poursuivre dans la mesure où les réseaux de transports sont de plus en plus saturés et du fait des gains de temps que permet ce type de véhicule. Une des pratiques les plus couramment adoptées est la « remontée de files », consistant pour le motocycliste à circuler entre deux files de véhicules. Malgré son interdiction dans plusieurs pays (États-Unis ${ }^{1}$, Allemagne...), cette pratique est aujourd'hui usuelle, et souvent tolérée, dans nombre de villes européennes et dans l'agglomération parisienne, particulièrement congestionnée. Notre recherche propose de mieux connaitre cette pratique telle qu'elle se déroule dans le trafic réel.

1 Excepté l'État de Californie où la remontée de files est tolérée. 


\section{1.- État de l'art}

La littérature scientifique disponible concerne l'aspect accidentogène de la circulation entre files et les motivations des conducteurs pour cette pratique.

\subsection{1.- Caractère accidentogène de la circulation entre files}

L'élément majeur qui ressort des études d'accidentologie est la faible implication de la pratique de l'inter-files dans la survenue des accidents (corporels/mortels) que ce soit sur le plan national ou international. Dans le rapport RIDER (Recherche sur les accidents Impliquant un DEux Roues motorisé, 2005), l'étude de 360 accidents se déroulant en France et impliquant un conducteur de deux-roues à moteur montre que 4,4\% des accidents concernent la circulation entre files. L'analyse des fichiers BAAC (Bulletins d'Analyse des Accidents Corporels, 2009) indique que la proportion des motocyclettes en position «entre deux files " impliquées dans des accidents représente en région parisienne $7,3 \%$ des accidents corporels et 4,5\% des accidents mortels. La surreprésentation en Ile-de-France semble liée (1) à la forte population de motocyclettes dans l'espace francilien, et (2) à la pratique même de la circulation entre files à Paris, plus répandue que dans les autres villes, et donc à une exposition au risque plus importante.

$\mathrm{Au}$ niveau européen, le projet MAIDS (Motorcycle Accidents In Depth Study, 2003) répertoriant les accidents qui se sont déroulés sur la période 1999-2000 dans cinq pays (France, Allemagne, Italie, Pays-Bas, Espagne) indique que 0,4\% des accidents impliquant un 2RM peuvent être liés à la circulation entre files (4 accidents sur les 921 pris en compte). Clarke, Ward, Bartle et Truman (2004) montrent que cette pratique est mise en cause dans $5 \%$ des accidents au Royaume-Uni. Crundall, Clarke, Ward et Bartle (2008) ont établi des statistiques sur ces accidents: ils concernent les remontées de files qui se déroulent en semaine (du lundi au vendredi) avec deux pics d'accidentologie à 8 heures et 17 heures, ce qui correspond, sans surprise, aux périodes de forte densité d'usagers (deux et quatre roues). En résumé, l'analyse des rapports d'accidents montre que la circulation entre files de la part des motocyclistes est impliquée entre 1 et $5 \%$ des accidents selon les zones géographiques considérées.

\subsection{2.- Motivations pour la pratique de la conduite entre files}

D'après la littérature, les bénéfices liés à la circulation entre files sont :

— la réduction de la congestion des agglomérations (Hurt, Ouellet, \& Thom, 1981 ; Sperley, \& Pietz, 2010). La réduction de l'occupation des files quatre-roues provient, pour une même demande de déplacement, du report modal d'une partie des autos vers les 2RM,

— la diminution du temps de trajet, qui est estimée comme la principale raison pour laquelle les conducteurs circulent entre les files (Hurt, et al., 1981 ; Projet Dft, 2004 ; Burge, Fox, Kouwenhoven, Rohr, \& Wigan, 2007). Pour Ellis (2006), la pratique de la conduite moto dans les agglomérations (intégrant la circulation entre files) permet de réduire de $50 \%$ le temps de trajet, comparé à une automobile. Dans Van Belleghem et Bourgeois (2004), la conduite entre files représente un gain de temps significatif pour les coursiers en 2RM, dans une activité soumise à des contraintes temporelles fortes. Pour les entreprises de courses, c'est en cela que l'intérêt du «deux-roues» réside par rapport au «quatre roues » selon ces derniers auteurs,

- la diminution des émissions de $\mathrm{CO}_{2}$, en relation avec la réduction du temps de trajet et malgré une optimisation moindre des 2RM comparé aux voitures (Ducreux, 2008),

- l'amélioration de la sécurité des usagers de la route. Hurt, Ouellet et Thom (1981) ont montré que la pratique de la remontée de files permettait d'augmenter les marges de sécurité entre les usagers. Selon la NHTSA (2000), la conduite entre les files dans un trafic arrêté ou en mouvement sur des voies multiples réduit la fréquence des accidents comparés à une conduite classique où les $2 \mathrm{RM}$ resteraient dans les lignes de circulation. 


\subsection{3.- Bilan de la littérature scientifique sur les remontées de files}

La littérature qui vient d'être évoquée apporte des éléments de connaissance intéressants sur les risques de la conduite entre files, les circonstances des accidents, et sur les principales raisons qui poussent les conducteurs à adopter ce type de pratique. Ces recherches montrent le faible pourcentage d'accidents en relation avec l'inter-files. Toutefois, il semble qu'il existe dans les situations de remontées de file un certain nombre d'événements ayant trait à la sécurité et/ou confort des conducteurs qui ne figurent pas dans les registres d'accidents. Il s'agit (1) de toutes les collisions qui ne nécessitent pas l'intervention de la police et pour lesquelles les forces de l'ordre ne dressent pas de procès-verbaux, et (2) des situations de «presque chute», qui sont l'ensemble des situations de conduite «rattrapées » de justesse par les usagers avant la collision, qui ne sont par définition pas répertoriées. Ces deux types de situations apparaissent toutefois être relativement fréquentes au regard de la rareté des accidents lors de la circulation entre files. Pour Sperley et Pietz (2010), on ne dispose pas de données suffisamment approfondies sur les comportements de conduite lors des remontées de files pour pouvoir comprendre ce que font réellement les conducteurs de 2RM et proposer des contre-mesures améliorant la sécurité. Ces limites peuvent, à notre avis, être dépassées en s'inspirant d'une approche d'orientation ergonomique.

\section{2.- Positionnement théorique}

Notre recherche s'inscrit dans la tradition des études d'ergonomie de langue française (Leplat, 1990 ; Theureau, 2006) où l'activité déployée par les acteurs en milieu «naturel» est au cœur des préoccupations. La situation «naturelle » est une situation non contrôlée par l'observateur, du point de vue des buts à accomplir, de la tâche à réaliser, de l'aménagement du milieu... C'est une situation que l'on peut qualifier «d'intacte». Un effort de réduction des perturbations occasionnées par la présence du chercheur, par la prise de vue, est réalisé de façon à s'assurer que le dispositif trouble le moins possible la tâche. Cet effort consiste en deux démarches : la discrétion des dispositifs d'observation et la précision des garanties pour les acteurs (anonymat, confidentialité) dans des contrats de collaboration. Toute une série d'études a été développée dans cette approche et a montré sa fécondité pour rendre compte de l'activité des individus dans des situations de vie quotidienne (Lave, 1988), de travail (Theureau, \& Jeffroy, 1994), sportive (Sève, Bourbousson, Poizat, \& Saury, 2009), de conduite automobile (Ciaccabue, \& Saad, 2008; Villame, 2004) et de conduite moto (Aupetit, Riff, Buttelli, \& Espié, 2011 ; Aupetit, Riff, Espié, \& Buttelli, 2009). La notion au cœur de cette approche est celle «d'activité » considérée comme une totalité intégrant les focalisations, attentions, perceptions, actions, communications et interprétations du conducteur. La démarche s'appuie sur la combinaison du recueil de données objectives (sur le comportement des acteurs) et des données subjectives (en relation avec leur expérience). L'approche utilisée permet de mener des études intégratives, c'est-à-dire qui décrivent la globalité de l'activité du conducteur (Saad, \& Villame, 1996).

L'objectif de la présente étude est ainsi d'analyser l'activité réellement mise en œuvre par les motocyclistes lors de la circulation entre files. Il s'agit de répondre aux questions suivantes : que font les motards lorsqu'ils remontent les files? Quel est le poids de la conduite entre files dans leur activité de conduite globale ? Quels sont les organisateurs de la conduite ? Sur quels éléments de la situation les motards se focalisent-ils, c'est-à-dire quels sont les changements dans leur champ attentionnel qui sont significatifs pour eux (Theureau, \& Jeffroy, 1994)? 


\section{2.- Démarche et méthodes}

\section{1.- Population}

11 motocyclistes ont été recrutés pour notre étude ${ }^{2}$ (Tableau 1). En raison des contraintes liées aux assurances pour l'autorisation de la conduite des motos instrumentées (Honda CBF 1000 et 800 VFR), la population d'étude comporte exclusivement des conducteurs expérimentés. En effet, les participants devaient détenir le permis A (moto) depuis plus de deux ans, ne pas avoir eu de suspension de permis au cours des deux dernières années, et posséder un véhicule de cylindrée équivalente ${ }^{3}$.

\begin{tabular}{|c|c|c|c|c|c|c|}
\hline Participants & $\begin{array}{l}\text { Hommes/ } \\
\text { Femmes }\end{array}$ & Âge & $\begin{array}{c}\text { Ancienneté } \\
\text { du permis } \\
\text { moto }\end{array}$ & $\begin{array}{l}\text { Moto conduite } \\
\text { habituellement }\end{array}$ & $\begin{array}{l}\text { Lieu de résidence } \\
\text { (département) }\end{array}$ & $\begin{array}{l}\text { Lieu de travail } \\
\text { (département) }\end{array}$ \\
\hline Motard 1 & $\mathrm{H}$ & 60 ans & 30 ans & Yamaha Fazer & Bures-sur-Yvette (91) & Paris $14^{\mathrm{eme}}(75)$ \\
\hline Motard 4 & $\mathrm{H}$ & 43 ans & 15 ans & BMW R1150RT & Collégien (91) & Paris $8^{\text {eme }}(75)$ \\
\hline Motard 5 & $\mathrm{H}$ & 29 ans & 13 ans & Suzuki 1100GSXR & Ecquevilly (78) & St Denis (93) \\
\hline Motard 6 & $\mathrm{~F}$ & 33 ans & 12 ans & Honda VFR 750 & Montlhéry (91) & Roissy (95) \\
\hline Motard 9 & $\mathrm{H}$ & 37 ans & 18 ans & Honda CBF 1000 & Corbeil-Essonnes (91) & Fleury-Mérogis (91) \\
\hline Motard 10 & $\mathrm{H}$ & 38 ans & 19 ans & Suzuki Bandit & Versailles (78) & Paris $16^{\text {eme }}(75)$ \\
\hline Motard 11 & $\mathrm{H}$ & 52 ans & 29 ans & BMW 1200RT & Gentilly (94) & Paris $15^{\mathrm{e} m e}(75)$ \\
\hline
\end{tabular}

Tableau 1 : Caractéristiques de la population étudiée

Table 1: Characteristics of the studied population

\section{2.- Recueil de données}

La démarche mise en place combine le recueil de données «objectives », du point de vue d'un observateur extérieur, et de données «subjectives", relatives à l'expérience des motards.

\subsection{1.- Données « objectives»}

Les données «objectives » sont recueillies à partir des deux motocyclettes instrumentées en caméras : une routière, Honda $1000 \mathrm{CBF}$, et une sportive/routière, Honda 800 VFR. Ces motos ont été prêtées pendant un mois aux participants qui ont réalisés leurs trajets habituels (domicile/travail et/ou loisirs). Des efforts ont été produits pour réduire au maximum la taille de l'instrumentation et pour la rendre la moins «intrusive» et visible possible. L'instrumentation n'a pas provoqué de modification des propriétés des véhicules (homologation conservée). Les enregistrements vidéo ont été réalisés à partir de quatre caméras embarquées (Figure 1). Trois caméras permettent de couvrir 160 degrés du champ visuel avant. Une quatrième caméra filme la face du conducteur (ou son casque) et permet d'appréhender les contrôles visuels. Au-delà de l'observation des comportements, ces enregistrements permettent de contextualiser a posteriori les situations de conduite et d'autoriser la tenue d'entretiens d'auto-confrontation ${ }^{4}$. Le dispositif d'enregistrement est situé dans le coffre arrière et les données sont stockées sur des cartes mémoire SD. Compte tenu des difficultés techniques rencontrées lors de cette étude «pilote» en Europe (nondémarrage de l'enregistreur, perte de vidéos en cours d'acquisition...), 148 trajets ont été enregistrés en vidéo, soit $25 \%$ des trajets réalisés par les motards pendant la période d'étude.

2 Cette étude a, pour partie, bénéficié du recrutement réalisé dans les projets DAMOTO (Détection de situations d'accidents en motocycles, 2009-2011) et 2BESAFE (Two wheeler behaviour and safety, 20092011).

3 Nous avons tenu compte de ces contraintes en termes de population d'étude dans l'analyse des données et la discussion.

4 Voir Rix et Biache (2004) pour une réflexion approfondie sur la réalisation d'entretien ayant comme support des vidéos recueillies avec caméras embarqués sur l'acteur. 
L'analyse se centre sur ces trajets enregistrés, ce qui correspond à 85 heures de vidéo.

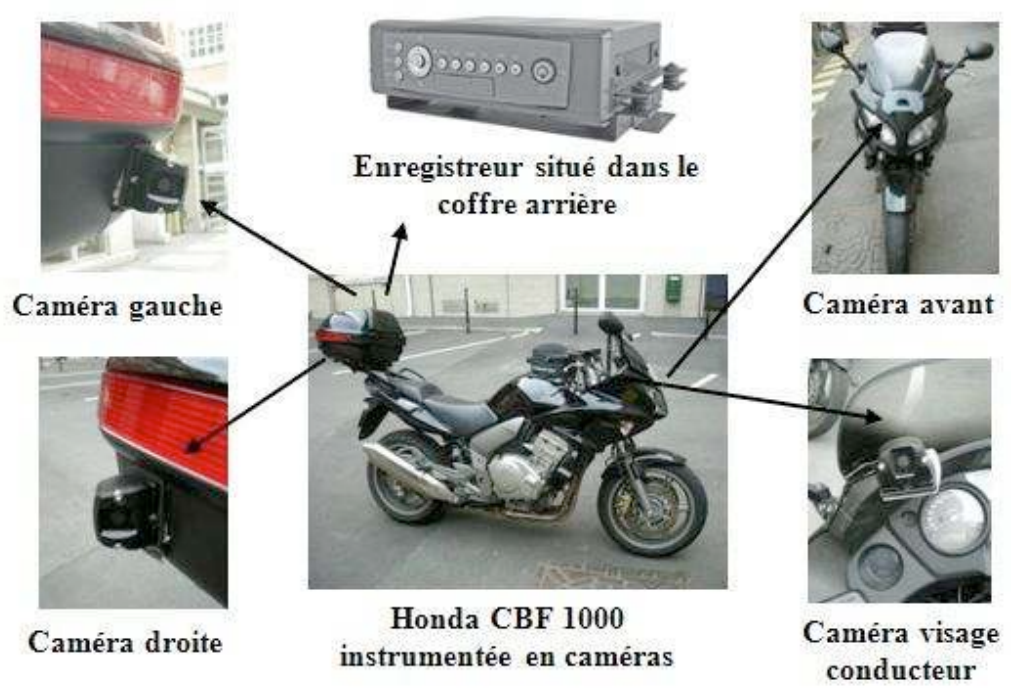

Figure 1 : Emplacement des quatre caméras embarquées et de l'enregistreur sur

le véhicule équipé

Figure 1: Camera instrumentation and video logger mounted on the equipped vehicle

Une mise en forme des données vidéo a été réalisée afin de permettre la tenue des entretiens. Cette mise en forme consiste à un positionnement des quatre vues afin d'être au plus près du contexte de trafic rencontré par le motard (Figure 2).

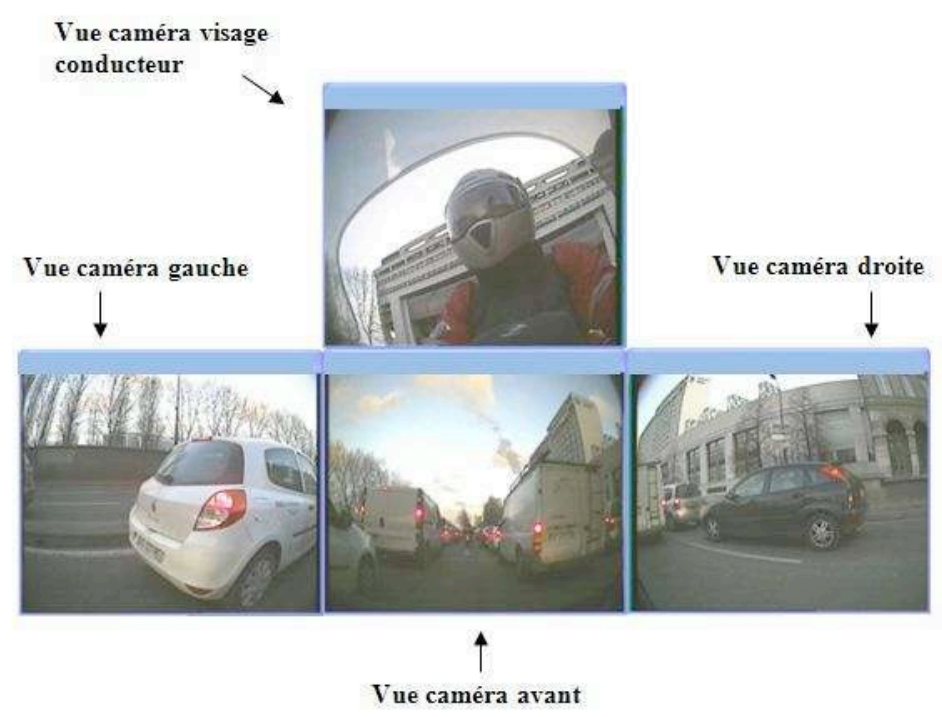

Figure $2:$ Mise en forme des vidéos pour la tenue des entretiens

Figure 2: Video browser interface for interviews

\subsection{2.- Données « subjectives »}

Les données subjectives ont été collectées au moyen d'entretiens d'auto-confrontation. Cette technique permet de décrire l'activité d'un individu, c'est-à-dire de reconstruire, à chaque moment, les mécanismes décisionnels et perceptifs utilisés, face à des traces vidéos de son propre comportement (Theureau, 2006). Testée dans Aupetit, Riff, Espié et Buttelli (2009) et Aupetit, Riff, Buttelli et Espié (2011), l'utilisation conjointe d'observations du 
comportement du motard et d'entretiens centrés sur l'explicitation «pas à pas » de son expérience offre des perspectives intéressantes pour l'étude de l'activité réelle de conduite intégrant le point de vue du motocycliste. Des épisodes vidéo de remontées de files recueillis en session de conduite sont projetés au motard (telles que présentées dans la Figure 3) qui les commente avec l'aide du chercheur. Les relances sont «centrées » sur l'action, afin que le motard puisse se replacer dans le contexte dynamique de la situation vécue. Les données collectées sont enregistrées avec le même matériel qui est utilisé pour recueillir les vidéos avec les véhicules instrumentés. Chaque entretien correspond à l'étude d'un trajet d'un motard (un déplacement «domicile/travail » par exemple) qui intègre de la circulation entre files et qui se déroule dans les trois jours précédant l'entretien (datation jugée suffisamment proche de l'entretien pour un niveau acceptable de mémorisation après plusieurs essais). La tenue des entretiens a été dépendante du bon enregistrement des vidéos. 31 entretiens ont été effectués (pour une durée totale de $13 \mathrm{~h} \mathrm{30}$ ), ce qui correspond à deux ou trois entretiens par participant selon la quantité de vidéos recueillies. La durée moyenne de ces entretiens est de 25 minutes.

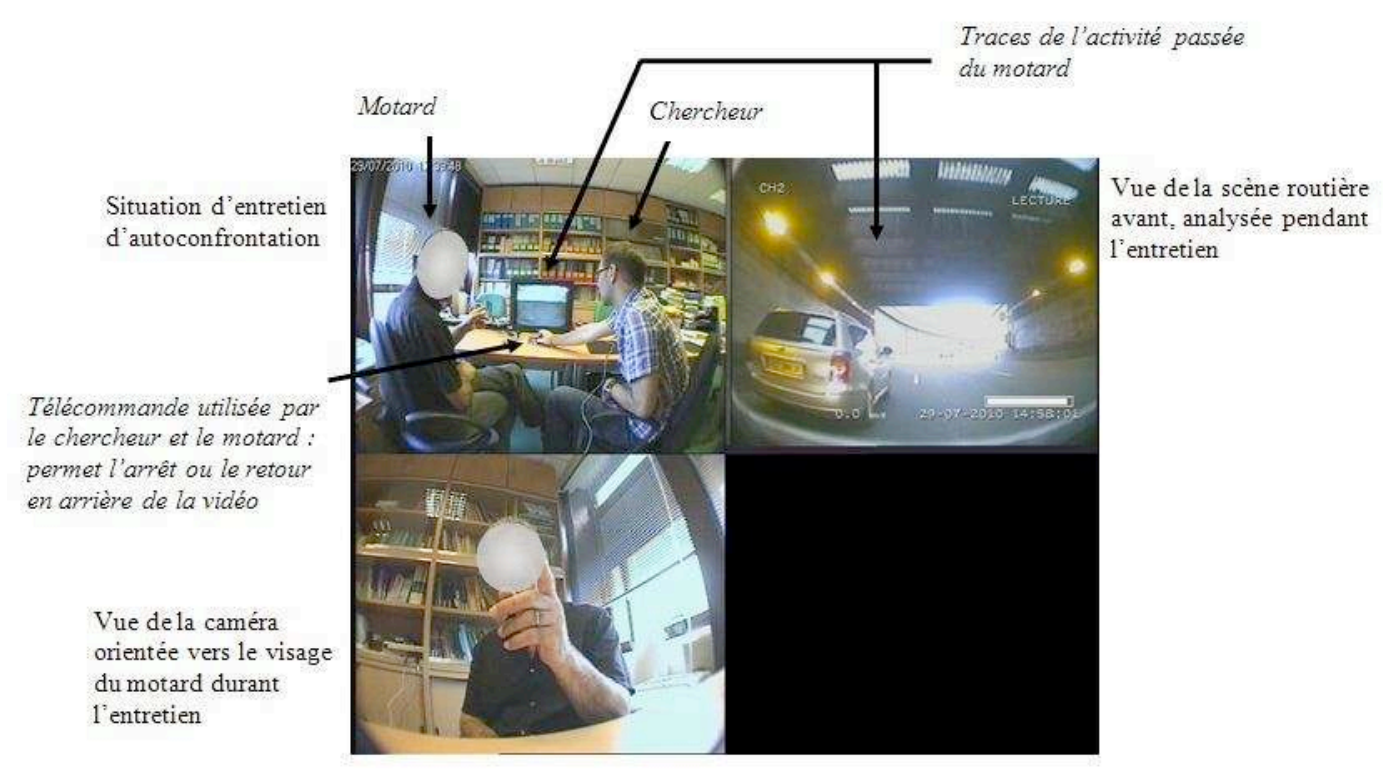

Figure 3 : Exemple d'une situation d'entretien d'auto-confrontation présentant la disposition des acteurs (motard/chercheur) et le matériel utilisé

Figure 3: Example of a self-confrontation interview situation showing the positioning of the participants (motorcyclist/researcher) and the equipment

\section{3.- Mise en forme et analyse des données}

Une grille de traitement combinant les données vidéo et les données verbales recueillies en entretien a été développée. Cette grille se compose de deux volets. Dans le premier sont répertoriées les «données vidéo » recueillies à l'aide des caméras embarquées. Ce volet est lui-même divisé en cinq grands ensembles : Temps (moment de la situation observée), Type de route (lieu de la situation de conduite observée), Infrastructure (infrastructure rencontrée par le motard), Comportement du conducteur de 2RM (actions, emplacement de l'interfiles...), État du trafic quatre roues (estimation de la densité du trafic, de sa vitesse instantanée, de l'espace inter-files). Est associé à ce premier volet, un second volet appelé « données d'entretien » comprenant trois ensembles : Temps (moment de l'entretien), Acteur (personne qui prend la parole), Verbalisations (retranscription verbatim de ce qui est dit pendant l'entretien). L'objectif est de synchroniser les deux volets de façon à ce que les verbalisations précisent le comportement observé.

Les catégories d'analyse ont été déterminées sur le modèle «top-down » (raisonnement 
déductif «classique») et «bottom-up» (raisonnement inductif). Elles sont le produit conjugué des questions de recherches (par exemple, une catégorie d'analyse porte sur le poids de l'inter-files dans la globalité de la conduite) et d'une première analyse des données collectées, faisant émerger des aspects significatifs de l'activité des motards. Le fonctionnement «bottom-up » a ainsi permis de créer des catégories d'analyse portant sur le différentiel de vitesse des motocyclistes avec le trafic ou sur les stratégies de recherche d'informations dans la situation de conduite qui sont apparues déterminantes pour les participants. L'immersion dans les données empiriques sert de point de départ au développement des catégories d'analyse et permet de conserver le lien avec les données de terrain (Glaser \& Strauss, 1967). L'intérêt de ce type de démarche est d'articuler les différents niveaux de données au sein d'une même structure de traitement et ainsi de pouvoir rendre compte, de manière synthétique, de l'intégralité des dynamiques d'activité.

\section{3.- Résultats et analyse}

Les résultats portent, dans une première partie, sur la description globale de l'activité réelle des motocyclistes lors la circulation entre files. Les données recueillies permettent de spécifier le poids de cette pratique dans les déplacements des motards et de décrire les types de vitesse qu'ils jugent adopter. La seconde partie se centre sur la description des focalisations des motards. Les données ont fait émerger l'existence de stratégies intensives de recherche d'informations dans la situation de conduite lors de l'inter-files. L'activité des participants semble systématiquement organisée autour de ces stratégies.

\section{1.- La description de l'activité réelle de circulation entre files}

\subsection{1.- Le poids de la pratique de l'inter-files dans la conduite des motocyclistes observés}

La totalité des 11 motards étudiés utilisant leur moto essentiellement pour réaliser des trajets «domicile/travail», l'analyse se centre sur ce type de déplacement. Le terme de trajet «domicile/travail», classiquement utilisé dans la littérature sur les transports, désigne les déplacements effectués par les conducteurs pour relier leur lieu de résidence à leur lieu de travail (Lyons \& Chatterjee, 2008). Les analyses des vidéos montrent que ce type de déplacement est systématiquement lié à des situations particulières du point de vue du contexte de trafic (forte densité d'usagers quatre-roues et 2RM aux heures de pointe) et de la dynamique interne du motard (trajet habituel, pression temporelle forte). La Figure 4 présente la durée et la distance moyenne parcourue en inter-files par trajet domicile/travail étudiées par rapport à la durée et la distance moyenne d'un trajet domicile/travail. Les données présentées sont issues de l'observation des vidéos de l'ensemble des déplacements de type « domicile/travail » des motards.

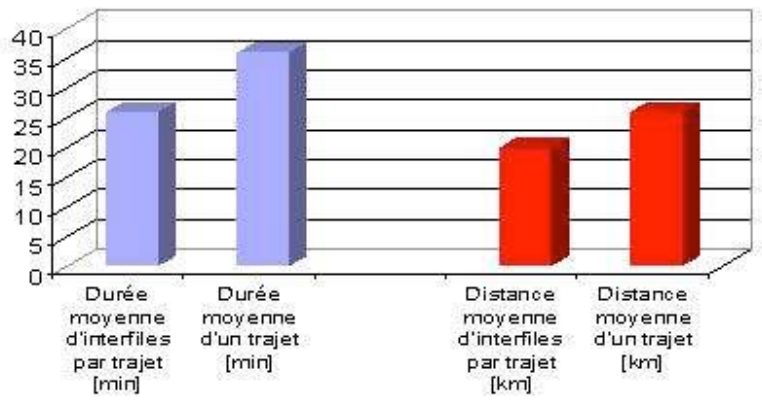

Figure 4 : Proportion de conduite en inter-files par trajet domicile/travail

Figure 4: Proportion of riding between traffic lanes per commute

Les données montrent que la circulation entre files est une activité systématique pour 
l'ensemble des participants : elle occupe en moyenne $72 \%$ du temps de conduite et environ $77 \%$ de la distance parcourue lors de chaque trajet domicile/travail. Ces chiffres illustrent le poids pris par ce type d'activité dans l'ensemble de la conduite des motards au cours de leurs trajets de ce type.

D'après les entretiens, la circulation en inter-files est pratiquée sur l'ensemble du réseau parisien (autoroute, périphérique, agglomération... 2x3 voies, $2 \times 2$ voies). Les motards ne conduisent pas en inter-files uniquement lorsque le trafic est fluide :

«Motard 11: On va arriver sur l'autoroute et je vais commencer à faire de l'inter-files.

Chercheur : Cette séquence d'inter-files, elle s'arrête à quel moment pour vous ?

Motard 11 : Sur ce trajet, je vais stopper de remonter les files juste avant d'arriver au travail, c'est-à-dire dans à peu près 45 minutes ! » (Entretien Motard 11)

«Motard 10 : Je suis en inter-files sur la totalité du trajet que j'emprunte tous les jours.

Chercheur : Dans le parcours que l'on regarde aujourd'hui...

Motard 10 : Dès que je suis entré sur l'autoroute c'était congestionné, je me mets direct en inter-files. Après j' arrive sur le périph et je continue de circuler en inter-files, jusqu'à mon travail dans le centre de Paris. » (Entretien Motard 10)

Les motocyclistes indiquent que la principale raison pour laquelle ils conduisent en interfiles est liée à la diminution du temps de parcours, par deux voire par trois, par rapport à la conduite d'une automobile :

«Sur mon trajet quotidien, je fais de la remontée de files dès que je suis sur l'autoroute et après sur le périph. Si ce n'était pas possible, je prendrais ma voiture. L'intérêt principal de la conduite en inter-files c'est le gain de temps. » (Entretien Motard 6)

« Tous les jours, je mets 30 minutes pour faire $30 \mathrm{~km}$ parce que je roule entre les files sur la quasi-totalité du parcours. Avant j'y allais en voiture, je mettais entre $1 \mathrm{~h} 15$ et $1 \mathrm{~h} 30$.» (Entretien Motard 4)

«Le trajet qu'on vient de visionner je l'ai fait en 55 minutes. Si je devais prendre ma voiture pour aller de l'Essonne jusqu'à l'aéroport Charles de Gaulle je mettrais facilement $2 \mathrm{~h}$ ou $2 \mathrm{~h} 30$ !» (Entretien Motard 11)

En résumé, les résultats montrent le poids et le caractère systématique de la pratique de circulation entre files pour l'ensemble des motocyclistes observés : une pratique quotidienne lors des trajets domicile/travail, qui s'étend sur l'ensemble du réseau étudié (autoroute, boulevard périphérique, agglomération... $2 \times 3$ voies, $2 \times 2$ voies...), et qui constitue la majeure partie des trajets des participants (la circulation entre files occupe en moyenne environ $3 / 4$ de chaque trajet domicile/travail).

\subsection{2.- Le différentiel de vitesse avec les voitures comme organisateur de l'activité}

Le premier élément, qui est commun à l'ensemble des motocyclistes observés, porte sur les modes de caractérisation de l'allure des motocyclistes. Chaque motard s'exprime en termes de différentiel d'allure (vitesse relative) avec les automobilistes et non de vitesse absolue. La variation de vitesse de la part des motards interrogés apparait clairement en relation avec la variation de l'allure du trafic, et plus précisément des files adjacentes à l'inter-files. Le différentiel de vitesse avec les voitures est ainsi, d'après les entretiens, un organisateur fort de l'activité de conduite entre files.

De manière plus détaillée, les données recueillies permettent de montrer les différentiels de vitesse typiquement adoptés par les participants sur le boulevard périphérique parisien (Figure 5), infrastructure empruntée par tous nos conducteurs et permettant donc des comparaisons. Les données présentées ici sont uniquement déclaratives. Pour la totalité des motards interviewés, il existe trois types de conditions de trafic, qui sont chacun associés à des classes de différentiels de vitesse avec les automobilistes: lorsque les files sont complètement arrêtées, lorsque les véhicules roulent à faible allure, et lorsque le trafic 
évolue à plus de $50 \mathrm{~km} / \mathrm{h}$.

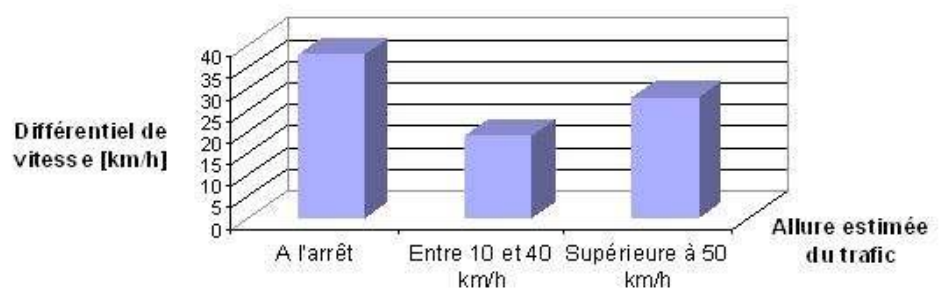

Figure 5 : Différentiel moyen de vitesse adopté par les motocyclistes sur le boulevard périphérique en fonction de l'allure du trafic d'après les entretiens

Figure 5: Average speed differential adopted by the riders on the Paris ring road compared to traffic speed, according to interviews

Lorsque le trafic est arrêté, la remontée de files s'effectue à $38 \mathrm{~km} / \mathrm{h}$ en moyenne d'après les conducteurs étudiés. Pour ces derniers, c'est une situation relativement "sûre », car les possibilités de changements de voies de la part des autres usagers sont rares :

«Pour moi c'est la situation la plus sûre pour remonter les files ! Lorsque les véhicules sont arrêtés, ils ne peuvent pas changer de voie. Je fais attention aux voitures de chaque côté, mais sans plus. » (Entretien Motard 8)

«Le trafic est arrêté et je me positionne à une allure satisfaisante. J'ai remarqué que j'étais tout le temps autour de $40 \mathrm{~km} / \mathrm{h}$. Cette situation ne représente pas trop de risques, les changements de voies sont rares. » (Entretien Motard 5)

« Je dois être à $40 \mathrm{~km} / \mathrm{h}$. Je ne regarde pas le compteur, c'est ma vitesse habituelle quand les voitures sont à l'arrêt. Les voitures ne peuvent pas changer de files, elles sont trop près les unes des autres. Je ne cours pas de risque.» (Entretien Motard 3)

En revanche, la plupart des motocyclistes déclarent qu'au-delà de $50 \mathrm{~km} / \mathrm{h}$ de différentiel avec les files de conducteurs automobiles arrêtées, ils ne se sentent pas en sécurité :

«Au-delà de $50 \mathrm{~km} / \mathrm{h}$ de différentiel de vitesse avec les files de voitures à l'arrêt, je ne me sens pas à l'aise, pas en sécurité, parce que les voitures défilent trop vite et je n'ai pas le temps de les surveiller assez attentivement. » (Entretien Motard 7)

«Si le différentiel de vitesse est autour de $30 \mathrm{~km} / \mathrm{h}$ ou $40 \mathrm{~km} / \mathrm{h}$ lorsque les voitures sont arrêtées je me sens en sécurité. Au-delà de $50 \mathrm{~km} / \mathrm{h}$, ce n'est pas possible pour moi d'être suffisamment attentif aux voitures. » (Entretien Motard 1)

«En fait quand les voitures ne roulent pas, je me sens bien jusqu'à $40 \mathrm{~km} / \mathrm{h}$. Après ça va trop vite pour moi. Le truc qui me fait peur c'est le mec qui déboite au dernier moment. » (Entretien motard 8)

«J'ai l'impression qu'à $40 \mathrm{~km} / \mathrm{h}$ tu peux mettre un bon coup de patin et freiner, mais pas à $50 \mathrm{~km} / \mathrm{h}$. J'ai aussi l'impression qu'à $40 \mathrm{~km} / \mathrm{h}$ de différentiel de vitesse avec le trafic arrêté, le choc sera moins important qu'à $50 \mathrm{~km} / \mathrm{h}$ ! » (Entretien Motard 3 )

Lorsque la vitesse du trafic est comprise entre 10 et $40 \mathrm{~km} / \mathrm{h}$, on note une réduction du différentiel d'allures moto/trafic d'après les entretiens (en moyenne $19 \mathrm{~km} / \mathrm{h}$ ). Les participants considèrent cet état particulier du trafic comme une situation problématique pour la conduite en inter-files, les changements de voies des automobilistes étant fréquents, rapides et, pour beaucoup, réalisés sans signalisation :

«Quand les voitures sont arrêtées et qu'il n'y a pas de trou, c'est-à-dire qu'elles ne sont pas tentées de changer de files, ça va. Par contre quand les files redémarrent c'est là qu'il faut faire attention parce qu'il y a des trous et les voitures changent souvent de voie sans clignotant en catastrophe. » (Entretien Motard 2) 


\section{«Chercheur : Qu'est-ce que tu fais là ?}

Motard 6: Je réduis ma vitesse parce que je vois que les automobilistes recommencent à rouler. C'est une situation vraiment très piégeante quand on remonte les files! (Entretien Motard 6)

«Là je fais très attention lorsque le trafic roule au pas. Je me suis fait des peurs avec des automobilistes qui changeaient au dernier moment de direction sans mettre le clignotant et sans regarder. C'est vraiment la situation aussi bien sur le périph que sur autoroute où on peut se retrouver par terre! » (Entretien Motard 7)

«Ce qui est dangereux c'est en dessous 40/50 km/h. Quand ça recommence à rouler, les gens sont énervés et veulent aller plus vite alors ils tentent de déboiter et souvent au dernier moment. Je suis beaucoup sur les freins et vachement attentif quand le trafic roule à $20 \mathrm{ou}$ $30 \mathrm{~km} / \mathrm{h} . »$ (Entretien Motard 9)

Lorsque la vitesse du trafic se situe au-delà de $50 \mathrm{~km} / \mathrm{h}$, différentes réactions ont pu être observées :

soit poursuivre la remontée de files avec un différentiel d'environ $28 \mathrm{~km} / \mathrm{h}$ par rapport au trafic environnant (pour 7 participants) :

«Chercheur : Là les voitures roulent bien et tu continues de circuler en inter-files...

Motard 9 : Oui tout le temps. Là on est sur le périph mais sur autoroute c'est la même chose. Peu importe l'allure des automobilistes, je fais de l'inter-files entre 20 et $30 \mathrm{~km} / \mathrm{h}$ au-dessus de leur vitesse. » (Entretien motard 6)

«Je suis à 130 ou $140 \mathrm{~km} / \mathrm{h}$ sur autoroute en inter-files lorsque le trafic est limité à $110 \mathrm{~km} / \mathrm{h}$, mais ça n'arrive presque jamais aux heures où je roule que le trafic soit si fluide. Sur le périph, je roule vers $100 \mathrm{~km} / \mathrm{h}$ quand ça roule bien. » (Entretien Motard 9)

« Je suis placé entre les deux voies de gauche et je me rends compte que c'est ma place! Je fais tout le temps de l'inter-files même quand le trafic n'est pas très important. Je roule tout le temps à $30 / 35 \mathrm{~km} / \mathrm{h}$ de plus que les voitures. » (Entretien Motard 4)

soit arrêter l'inter-files (pour 4 participants) :

«Quand les files de voitures roulent à 70 ou $80 \mathrm{~km} / \mathrm{h}$, si je fais de l'inter-file, je vais devoir rouler à $100 \mathrm{~km} / \mathrm{h}$. Faire de la remontée de files à $50 \mathrm{~km} / \mathrm{h}$, déjà en cas de choc tu es mal, alors à $100 \mathrm{~km} / \mathrm{h}$ ! (Entretien motard 5)

«Quand ça roule bien je ne gagne pas de temps si je fais de l'inter-files lorsque les voitures roulent bien donc je reste derrière elles. Au niveau temps de trajet, ça ne change pas grandchose. » (Entretien Motard 1)

« Je m'arrange pour ne pas descendre en dessous de $50 \mathrm{~km} / \mathrm{h}$. Quand les voitures n'avancent plus, je conserve $50 \mathrm{~km} / \mathrm{h}$. Quand les voitures dépassent $50 \mathrm{~km} / \mathrm{h}$, je ne dépasse plus les files, je roule comme les voitures.» (Entretien motard 10)

«Ma hantise c'est que quelqu'un déboîte. À $50 \mathrm{~km} / \mathrm{h}$, si c'est pas trop surprenant, je pense que je peux m'en sortir. Au-delà de $50 \mathrm{~km} / \mathrm{h}$ de différence avec les voitures je ne me sens pas capable. » (Entretien Motard 4)

$\mathrm{Au}$ bilan, les entretiens ont permis de mettre en évidence les relations existantes entre la vitesse adoptée par les motards. Ce qu'il faut retenir est que la situation jugée comme la plus sûre est la situation où le trafic est arrêté : la distance inter-véhicules est si faible que les changements de voies des automobilistes sont très rares. Lorsque le trafic se remet en marche, la situation est jugée par les motards comme très risquée puisque les changements de voies sans signalisation sont très fréquents. Le différentiel moyen de vitesses dans l'interfiles est alors réduit, aux alentours de $19 \mathrm{~km} / \mathrm{h}$. 


\section{2.- Étude des focalisations des motards lors de la circulation en inter- files}

Les observations de l'activité de circulation inter-files associées aux entretiens menés $a$ posteriori font émerger l'existence d'une recherche intensive d'informations dans la situation de conduite pour l'ensemble des participants. Les focalisations des motards s'orientent vers un certain nombre d'informations hétérogènes qui sont présentées ci-après. La manière dont ces focalisations s'organisent dans la situation pour les conducteurs observés sera ensuite abordée.

\subsection{1.- L'orientation des focalisations des motards lors de l'inter-files}

\section{a) Vers l'état global du trafic}

D'après les données récoltées, l'espace inter-files, c'est-à-dire la largeur du couloir existant entre les deux files de voitures que remonte le motard, fait l'objet de focalisations systématiques. Cet espace inter-files est impliqué dans la quasi-totalité des décisions d'actions des conducteurs :

«Ce que je regarde en premier c'est la largeur que me laissent les voitures devant, si j'ai la place pour passer. Dans la situation présentée à la vidéo, j'ai la place donc je m'engage. » (Entretien Motard 8)

«Pour moi, ce qui va faire que tu vas arrêter ou poursuivre l'inter-files c'est avant la largeur de l'entre files. » (Entretien Motard 2)

«Évidemment il y a beaucoup d'éléments qui vont faire que je vais diminuer ma vitesse et arrêter de faire de l'inter-files, mais le principal c'est que le couloir pour passer n'est pas suffisamment grand pour que je continue de rouler entre les files » (Motard 11)

«Chercheur : À quoi tu fais attention dans cette situation ?

Motard 9 : Je vois que j'ai la place pour passer, c'est ce que je regarde en premier...

Chercheur : Comment voyez-vous que vous avez la place pour passer ?

Motard 9 : Je l'estime par rapport à la taille de mon guidon, si je sens que mon guidon passe dans le couloir, c'est que je peux passer. » (Entretien Motard 9)

On a démontré plus haut que l'allure des conducteurs automobiles était également un paramètre important pris en compte par les motards. Pour ces derniers, il s'agit de conserver un différentiel de vitesse satisfaisant avec les automobilistes du point de vue de leur sécurité et leur confort. La figure suivante (Figure 6) illustre ce résultat. Elle représente l'analyse d'une situation de conduite en inter-files vécue par le participant 6 :
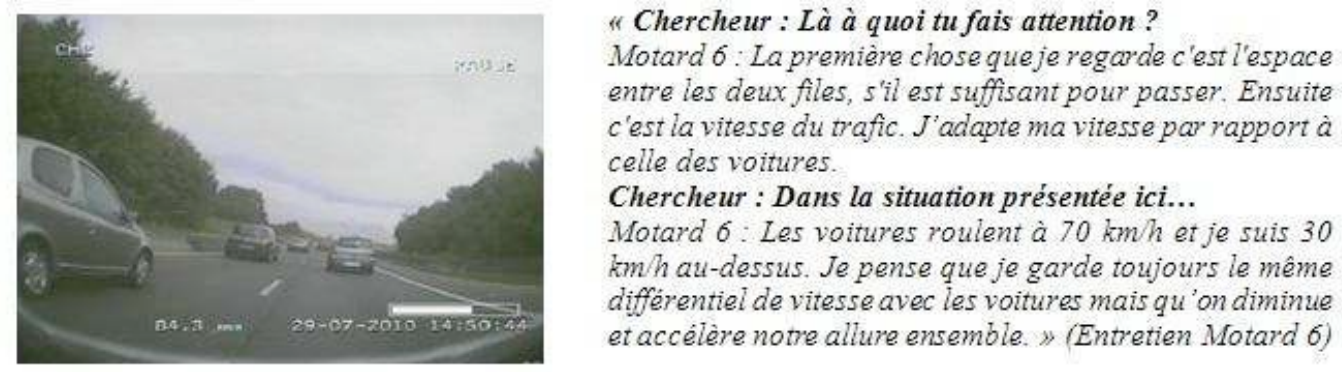

Figure 6 : Analyse d'une situation de conduite entre files du participant 6 avec entretien et vidéo

Figure 6: Analysis of a lane-splitting situation with rider 6 by interview and video

Le troisième élément significatif dans le trafic pour les motards est la distance intervéhicules dans les files adjacentes. Les données montrent que même si l'espace inter-files est faible, le fait que la distance inter-véhicules dans chaque voie soit importante laisse la 
possibilité aux motocyclistes de slalomer entre les véhicules pour remonter les files (Figure 7).
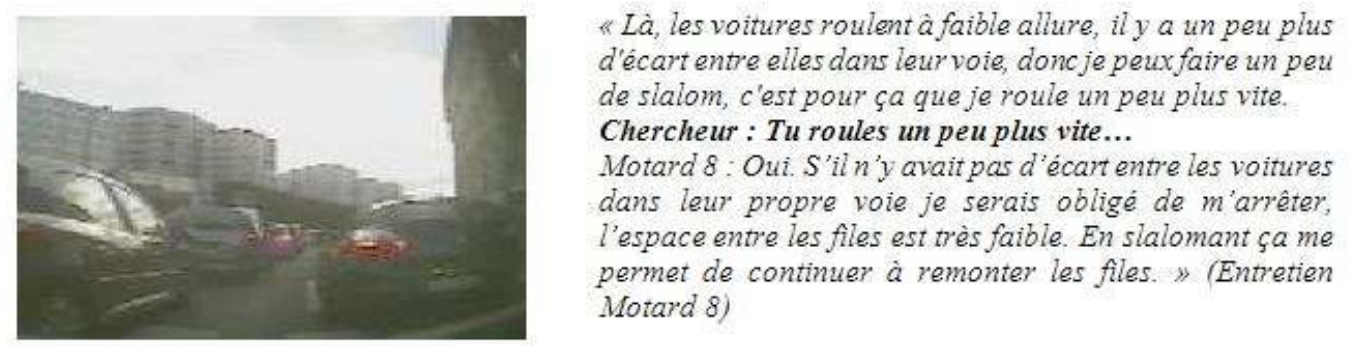

Figure 7 : Analyse d'une situation de conduite entre files du participant 8 avec entretien et vidéos

Figure 7: Analysis of a lane-splitting situation with rider 8 by interview and video

\section{b) En direction des comportements à risque des automobilistes}

Les entretiens indiquent que l'activité des motocyclistes est largement orientée par la détection d'informations précises dans le comportement des conducteurs automobiles qui pourraient traduire un potentiel comportement à risque (par exemple, un déplacement latéral du véhicule). Les focalisations des motards concernent principalement l'orientation des roues des véhicules, les gestes des conducteurs dans l'habitacle et la plaque d'immatriculation du véhicule indiquant l'origine géographique de l'automobiliste.

L'orientation des roues des automobiles. Une part importante de l'attention des motards semble focalisée sur le déplacement des automobilistes dans leur propre voie. Les entretiens montrent qu'à basse vitesse ils appréhendent principalement le déplacement des conducteurs à partir de l'orientation des roues :

«Chercheur : Tu t'engages dans la remontée de files, à quoi tu fais attention là ?

Motard 10: Là je fais surtout attention aux mouvements de roues des voitures. Pas les voitures juste devant moi, mais celles d'après. » (Entretien motard 3)

« Dès que l'on voit les roues qui sont tournées, ça veut dire que le conducteur veut changer de voie. Il vaut mieux quelques fois anticiper ces situations parce que certains conducteurs déboitent sans clignotant et sans regarder ! (Entretien Motard 10)

«Pour moi, le principal indice auquel je fais attention quand je remonte les files ce sont les roues des voitures. Si tu prends le véhicule dans son ensemble, tu ne peux savoir que le conducteur va déboiter. Les roues se sont les premiers éléments qui t’indiquent que la voiture va changer de files donc je fais très attention à cela. » (Entretien Motard 2)

"Chercheur : Comment tu as vu que la voiture de gauche voulait se rabattre?

Motard 9: Déjà j'ai vu que sa roue avant droite était tournée vers la file de droite donc j'ai fait attention. Après la voiture a mis son clignotant donc ça a confirmé qu'elle tournait à droite. » (Entretien Motard 9)

Les gestes des conducteurs dans l'habitacle. D'après les entretiens, les motocyclistes sont très attentifs à ce qui se passe dans l'habitacle des voitures à proximité. Ils recherchent des indices qui pourraient traduire l'inattention des conducteurs et provoquer un mouvement latéral. Tous les participants ont déclaré faire attention aux «écrans lumineux » des GPS et téléphones portables souvent utilisés par les conducteurs :

«Motard 1: Ce qui est dangereux quand on remonte les files se sont les conducteurs inattentifs ! On voit plein de conducteurs qui ont un comportement anormal, qui jouent avec leur téléphone, qui envoient des textos ou qui règlent leur GPS tout en conduisant.

Chercheur : Un comportement anormal?

Motard 1: Cela veut dire que les voitures vacillent dans leur voie et ont des vitesses 
irrégulières et différentes du trafic. » (Entretien Motard 1)

«Là je viens de ralentir parce que j'ai vu un grand écran lumineux dans la voiture et le mec qui se penche dessus ! Je m'en rappelle bien. L'espace entre les files n'est pas large et il suffit que cet automobiliste se déporte légèrement sur la gauche quand je passe à côté et je suis mort !» (Entretien Motard 8)

L'utilisation de ce type de dispositif tend, d'après les motocyclistes, à distraire les conducteurs non seulement par la manipulation, mais aussi par l'attention allouée. De plus, les motocyclistes interrogés apparaissent sensibles aux mouvements de tête des automobilistes en direction du siège passager dans l'habitacle. La plupart des motards disent avoir vécu des situations dangereuses pendant qu'ils circulaient entre les files à cause d'un déport latéral involontaire d'un conducteur automobile en pleine discussion avec son ou sa passagère :

«Quand je remonte les files, je fais très attention aux personnes qui sont dans les voitures, j'essaye notamment de voir si la tête de la personne n'est pas dirigée vers le passager. Dans ces cas-là, ça veut dire que le conducteur est en train de discuter et qu'il peut très bien se déporter, moi, donc je fais attention. » (Entretien Motard 3)

«Motard 10 : Quand l'allure le permet, je regarde dans les voitures.

Chercheur : Vous regardez quoi exactement ?

Motard 10: Je fais surtout attention aux personnes qui discutent. Le problème est que, comme on passe très près des voitures en inter-files, le moindre écart de la bagnole peut renverser le motard. » (Entretien Motard 10)

Le numéro de département présent sur les plaques d'immatriculation des automobilistes. D'après les données, la majorité des motards prête une grande attention à l'origine géographique des automobilistes par l'intermédiaire du numéro de département figurant sur les plaques d'immatriculation. Ce phénomène tend à s'estomper avec la possibilité récente d'opter pour le numéro de département de son choix. Un certain nombre d'extraits du corpus mettent en lumière cet indice comme un des indices principaux pris en compte pour s'engager ou non dans l'inter-files :

«Chercheur : Dans la situation présentée à la vidéo là, vous prêtez attention à quoi ?

Motard 5 : Je regarde les plaques d'immatriculation des voitures. Tous les motards vous le diront. Si je vois une plaque qui n'est pas de l'Île-de-France, je vais me méfier. Je fais attention à sa trajectoire. Après si c'est ok, je vais rouler doucement en passant à côté d'elle et être très prudent. » (Entretien Motard 5)

«Motard 7 : Le numéro de département est vraiment important quand je remonte les files. Dans le 95, il y a beaucoup de conducteurs du 27 ou du 60, et ils n'ont pas du tout le même comportement que les conducteurs parisiens.

Chercheur : Quelles différences existent ?

Motard 7 : Par exemple une voiture immatriculée dans le 60 ne va jamais se rabattre pour me laisser passer. » (Entretien Motard 7)

Si des éléments de la situation de conduite laissent à penser aux motards qu'ils pourraient courir un risque, ces derniers ralentissent de manière systématique et se déclare être « en état de vigilance » à l'égard du ou des véhicules concernés.

\section{c) Vers les autres $2 R M$}

Pour chaque motocycliste étudié, le comportement des autres usagers 2RM utilisant eux aussi l'inter-files est un des éléments de leur champ attentionnel les plus significatifs. Certaines catégories d'usagers 2RM sont considérées comme des catégories d'usagers à risque, dont il faut se méfier. C'est le cas des conducteurs de scooters de cylindrée de $125 \mathrm{~cm}^{3}$, autorisés à la conduite de cet engin grâce à leur permis B. Les motards indiquent que ces scootéristes adoptent typiquement des comportements à risque: ils freinent brusquement, accélèrent violemment et surtout ils n'ont pas le respect vis-à-vis des autres 
usagers de $2 \mathrm{RM}$, à la différence de ce qui existe entre motards :

«Les scooters qui ne font que de la ville n'ont pas le même comportement. Ceux qui ne sortent que l'été font les cow-boys! Ce sont des mecs que tu retrouves à l'hôpital!» (Entretien Motard 7)

« J'ai un gros problème avec les scooters! Je garde mes distances par rapport à eux. Ils ne respectent rien. Les mecs vont te doubler à gauche, à droite alors que tu remontes les files !» (Entretien Motard 11)

«Les vrais dangers aujourd'hui ce sont les scooters parce qu'ils ne conduisent pas comme des motos. Ce sont des automobilistes qui ont pris un deux roues et c'est infernal!» (Motard 2)

«Les scootéristes sont dangereux. Ils ne respectent pas les règles des motards : on se laisse dépasser, on roule en file. Eux ce sont des automobilistes et ils n'ont pas conscience du fonctionnement des deux roues! $\gg($ Entretien Motard 4)

Ces difficultés d'interactions semblent encore plus saillantes avec une autre catégorie d'usagers, ceux de maxi-scooters (véhicule $2 \mathrm{RM}$ dont la cylindrée est supérieure à $\left.500 \mathrm{~cm}^{3}\right)$ :

«Motard 13: Ah un maxi-scooter juste devant moi! Tous les motards vous le diront : les maxi-scooters ce sont des plaies! C'est vraiment une bande à part.

\section{Chercheur : Les maxi-scooters ce sont des plaies...}

Motard 13: Les conducteurs de maxi-scooter c'est affolant! C'est simple : les stops, ils ne connaissent pas! Les feux, même si le piéton est encore au vert, ils vont passer quand même ! Ils n'ont aucune règle. » (Entretien Motard 1)

«Sur le périph, le motard va respecter les autres, c'est-à-dire pas de klaxon quand on remonte les files; la route est à tout le monde. Le mec qui a un maxi-scooter, il arrive dans l'inter-files et il klaxonne, il fait des appels de phares! Comme si la route était à lui ! » (Entretien Motard 5)

«Le scooter devant, il accélère comme un fou, il freine, il accélère, il colle la moto devant de près ! Voilà c'est habituel avec les maxi-scooters ! (Entretien Motard 11)

Les observations montrent que les participants prennent un certain nombre de précautions quand ils approchent de tels usagers (scootéristes $125 \mathrm{~cm}^{3}$ et usagers de maxi-scooters) : réduction de l'allure, augmentation de la distance de sécurité... comme le montre le participant 8 dans la figure suivante (Figure 8).

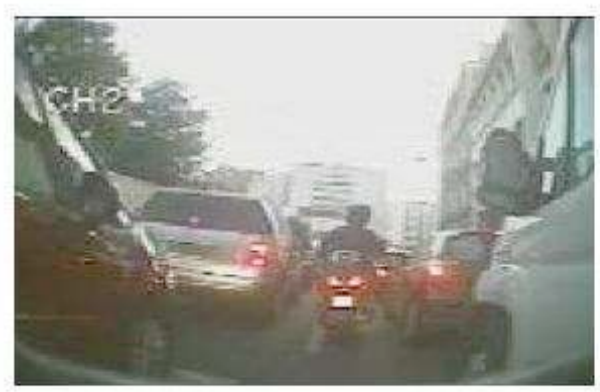

«Chercheur : Qu'est-ce qu'il se passe pour toi là? Motard 8. Je fais attention au maxi scooter devant. Pour moi c'est le facteur de risque le plus important ici. Chercheur : Le facteur de risque le plus important... Motard 8: J'ai remarqué qu'il avait une conduite pas très fiuide, il accélère, il freine brutalement. Je peux me le prendre s'il freine brusquement !

Chercheur: Qu'est-ce que tu fais toi?

Motard 8: Je vais réduire un peu ma vitesse et surtout garder mes distances par rapport à lui. Je ne vais pas le coller. " (Entretien Motard 8)

Figure 8 : Analyse d'une situation de conduite entre files du participant 8 avec entretien et vidéo

Figure 8: Analysis of a lane-splitting situation with rider 8 by interview and video

En plus de ces catégories d'usagers à risque déterminées a priori, les motards déclarent être «en alerte» par rapport à d'autres conducteurs de deux-roues motorisés qui pourraient présenter un risque dans la situation : 
«Là je fais super gaffe aux deux roues qui me précèdent parce que je vois bien qu'il accélère rapidement et freine aussi brutalement. C'est un fou! Donc je m'éloigne de lui quand je remonte. » (Entretien Motard 10)

«Je suis ce motard depuis quelque temps déjà et je me suis rendu compte qu'il avait une conduite dangereuse. Il passe très près des bagnoles, les évite au dernier moment. Il a une conduite plutôt agressive donc je ne le colle pas. » (Entretien Motard 11)

D'après les entretiens, l'activité des autres usagers 2RM dans l'inter-files influence ainsi grandement le comportement des motards. Les participants déclarent opter pour des allures qui leur évitent d'être gênés par les autres usagers 2RM qui remontent les files :

«Motard 1: Sur le périphérique, je roule habituellement autour de $40 \mathrm{~km} / \mathrm{h}$ quand les voitures sont à l'arrêt de façon à ne pas se faire embêter derrière.

\section{Chercheur : Embêter derrière...}

Motard 1: Par des deux-roues plus pressés que moi ! Au départ je roulais à $30 \mathrm{~km} / \mathrm{h}$ et je devais sans cesse me ranger, puis me réinsérer. Maintenant je ne me range plus très souvent, je pense 4 ou 5 fois sur l'ensemble du trajet. » (Entretien Motard 1)

« Je trouve que beaucoup de motards sont stressés. Quand je suis en tête de file, je sens qu'il y a des motards qui poussent derrière moi, qui aimeraient bien aller plus vite. À ce momentlà, je me rabats, je me remets entre deux bagnoles. Je refuse d'aller plus vite donc je les laisse passer. » (Entretien Motard 4)

« Motard 9 : Je m'insère dans le trafic et je me retrouve entre deux deux-roues.

\section{Chercheur : Qu'est-ce que tu fais à ce moment-là ?}

Motard 9: Je m'adapte par rapport à la vitesse des deux roues qui sont devant et derrière moi, de façon à ne pas obliger ceux derrière moi à freiner brusquement et ceux de devant à stresser ! » (Entretien Motard 9)

\section{d) En direction des zones d'infrastructure spécifiques}

Des infrastructures particulières reviennent systématiquement dans le discours des motocyclistes pour expliquer un changement de comportement lors des remontées de files. Par exemple, la présence d'insertions à proximité (entrée ou de sortie de voie) pousse le motard à ne pas engager d'inter-files ou à réduire sa vitesse, ces zones étant associées typiquement à des changements de voies fréquents :

«On arrive sur l'autoroute du Sud. C'est toujours des moments difficiles ces transitions entre deux autoroutes parce qu'il y a pas mal de changements de voies donc il faut être attentif. C'est comme toutes les intersections ou arrivées ou sorties de voie c'est très dangereux quand on remonte les files. » (Entretien Motard 6)

«Là je sais qu'il y a une voie à gauche que les gens prennent souvent donc je fais attention. C'est un cas d'accident ça en remontée de file lorsque des voitures coupent la voie pour aller sur la gauche. » (Entretien Motard 8)

« Il y a beaucoup de mouvements jusqu'au tunnel du Châtelet. Il faut faire très attention dans cette portion, car il y a des sorties à droite et à gauche. Il y a la route qui fait deux fois deux voies juste avant Châtelet, les véhicules vont se croiser et donc il faut être très prudent en remontée de files. » (Entretien Motard 9)

De la même manière, les données montrent que l'allure des motocyclistes se réduit en interfiles à l'approche de radars automatiques :

«Là je réduis ma vitesse dans l'inter-files à cause du radar que l'on va bientôt croiser. Je connais bien l'emplacement de celui-ci. » (Entretien Motard 7)

«Motard 2: Il faut faire attention ici parce qu'il y a un radar parce qu'il y a beaucoup de conducteurs qui freinent brusquement à proximité.

Chercheur : Qu'est-ce que tu fais toi à ce moment-là ?

Motard 2: Moi je vais réduire ma vitesse légèrement, attendre de passer le radar et ré 
accélérer ensuite pour revenir à ma vitesse de croisière. » (Entretien Motard 2)

\section{e) En relation avec les conditions météorologiques}

Pour la majorité des motards, des conditions météorologiques dégradées, comme la pluie, ont des conséquences importantes sur leur saillance visuelle vis-à-vis des autres usagers et sur le freinage. Ces conditions font que leur pratique de la remontée de files est moins intensive : c'est-à-dire moins rapide et avec moins de changements de couloir d'inter-files.

« Il pleut pas mal donc forcément les conditions de visibilité ne sont pas au top et je ne suis vraiment pas sûr que les voitures me voient bien. » (Entretien Motard 9)

« Il commence à pleuvoir donc je vais réduire ma vitesse de quelques $\mathrm{km} / \mathrm{h}$. On freine beaucoup moins bien sous la pluie. » (Entretien Motard 11)

«Là comme il pleut, les automobilistes me voient moins bien, et aussi l'adhérence est pas très bonne, je décide de ne pas me faufiler entre les deux voitures. » (Entretien Motard 10)

En résumé, les entretiens soulignent (1) le nombre important d'aspects de la situation qui font l'objet de focalisations systématiques de la part des motards, (2) leur importante diversité (cinq catégories différentes d'informations dans notre analyse), et (3) les relations entre focalisations et modalités d'actions du conducteur au cours de l'inter-files.

\subsection{2.- L'étude des processus de focalisations des motards entre les files}

Les données récoltées permettent de mettre en évidence le fait que les focalisations des motards s'orientent, de manière simultanée, vers plusieurs types d'informations pour s'engager et agir dans les remontées de files :

«Dans la situation là, je regarde l'espace que j'ai pour passer entre les voitures, je fais aussi attention à leur vitesse et je regarde s'il n'y a des roues tournées vers moi, synonyme que quelqu'un va déboiter. » (Entretien Motard 7)

« Je fais attention à deux choses lorsque je remonte les files : la largeur du couloir de l'interfiles devant moi et les entrées et sorties sur la route. » (Entretien Motard 3)

«Vous voyez le véhicule sur la droite, il commence à orienter ses roues vers l'inter-files donc je fais attention. Je regarde aussi l'espace entre les voitures s'il est suffisant pour moi et je fais gaffe au radar qui va bientôt arriver parce que les conducteurs ont tendance à freiner fort à son approche. » (Entretien Motard 5).

Les entretiens montrent que les dynamiques d'activité des motards dans les situations d'inter-files sont davantage en relation avec une combinaison d'informations de l'environnement plutôt qu'avec un type d'informations isolé. Plusieurs sources d'attention sont prises en compte quasi instantanément pour adapter les modalités d'actions en conséquence. Les données disponibles n’ont pas permis de relier des stratégies spécifiques de focalisations à des situations de route particulières. Toutefois, comme indiqué plus haut, les résultats indiquent que les focalisations des motards s'orientent massivement vers le dimensionnement de l'espace inter-files. Cette dimension étant récurrente lors de chaque décision d'action des motards. D'après les données, le motard «évalue » son gabarit (et celui de son véhicule) par rapport à la largeur du couloir existant entre les véhicules de chaque côté de l'inter-files. Plutôt que relevant d'une «comparaison» de gabarit, les données semblent montrer que le passage entre deux véhicules relève d'un ressenti, de quelque chose de plus abstrait :

\section{«Chercheur : Comment vous savez que vous passez entre ces deux voitures ?}

Motard 4 : Je le sais. J'ai le gabarit de la moto dans la tête et je sens que là je vais passer, même si quand on voit la vidéo c'est vrai que c'est vraiment très juste ! » (Entretien Motard 4)

« Motard 10 : Là je ne pensais pas du tout que je pouvais passer entre les voitures. Elles étaient très proches l'une de l'autre. Même après quelques semaines de pratique avec la 
moto je la connais déjà bien, je me suis familiarisé avec son gabarit.

Chercheur : C'est-à-dire...

Motard 10 : Je sais si je passe ou pas. Je le sens...»(Entretien Motard 10)

Les entretiens avec les motards semblent valider l'idée que le gabarit de leur propre véhicule est un élément incorporé, qui fait partie de leur schéma corporel (même après seulement quelques semaines de pratique avec la moto «expérimentale»). Il s'agit dans ce cas de considérer la frontière du corps du motard comme «flexible », c'est-à-dire pouvant s'étendre à son véhicule et à ces dispositifs de protection (casque, blouson...). Ce résultat mérite des investigations plus approfondies qui pourraient notamment se centrer sur les mécanismes d'incorporation dans les premières expériences de conduite des objets utilisés par le motard, et leurs relations avec l'apprentissage de la conduite.

Les données montrent également la forte distribution des informations dans l'ensemble de l'espace visuel du motocycliste, l'obligeant à porter son attention dans des directions différentes dans un décours temporel restreint :

«Chercheur : A quoi vous faites attention dans la situation à la vidéo ?

Motard 3 : Je fais attention bien sûr à la largeur du couloir juste devant moi. Je regarde aussi au loin pour voir s'il n'y a pas d'obstacle dans l'inter-files, si quelqu'un n'est pas en train de changer de voie. Et puis en même temps je regarde dans l'habitacle des voitures si les conducteurs ne sont pas en train d'utiliser leur téléphone portable ou leur GPS. » (Entretien Motard 3)

« Dans cette situation, je fais très attention à ce qui se passe dans les véhicules tout proches de moi, aux discussions et à l'utilisation de GPS. J'ai une très bonne vision périphérique, ça m'aide pour cela. En même temps, je suis capable de regarder au loin si les feux-stops des voitures ne s'allument pas. » (Entretien Motard 1)

« Je prête beaucoup d'attention au revêtement de la route quand je fais de l'inter-files. Évidemment je n'ai pas les yeux rivés vers le bitume, je suis également concentré sur les deux voitures de chaque côté de l'inter-files. Je regarde aussi à l'horizon pour anticiper si une voiture décide de changer de voie au dernier moment. » (Entretien Motard 9)

D'après les motards, la pratique qui consiste à porter son attention vers plusieurs points répartis dans la situation est facilement réalisable lorsque la vitesse n'est pas trop élevée, c'est-à-dire en dessous de $50 \mathrm{~km} / \mathrm{h}$. C'est le cas lorsque le trafic est arrêté sur le boulevard périphérique. C'est également une situation qui, du fait de la densité du trafic et des risques associés, nécessite une recherche approfondie d'informations combinées.

En synthèse, les motards observés semblent se focaliser sur des éléments précis de la situation de conduite et ce, de manière combinée, pour détecter les éventuelles situations à risque et agir dans les remontées de files. Même si l'espace inter-files fait l'objet d'une attention particulière et systématique, les focalisations des motocyclistes sont orientées dans plusieurs directions et les changements dans leur champ attentionnel semblent extrêmement rapides.

\section{4.- Discussion}

Les résultats de cette étude sont tout d'abord discutés sur le plan de la compréhension de la pratique de l'inter-files. L'analyse des focalisations apporte un éclairage original et permet de caractériser les motards étudiés en tant qu' «experts » de la lecture des situations de conduite entre files. Nos résultats sont également intéressants au plan de la conception. D'un côté, ils questionnent la possibilité d'une législation sur les remontées de files, et de l'autre, ils ont également des implications fortes pour l'apprentissage et la formation à la conduite. 


\section{1.- Les motards comme « experts » de la lecture de situations de conduite entre files}

Les résultats ont indiqué que les focalisations des motocyclistes expérimentés et utilisateurs de motos de grosse cylindrée que nous avons suivis portaient sur plusieurs éléments de la situation. Ces éléments concernent l'activité des autres usagers à l'intérieur (utilisation de téléphones portables ou GPS, mouvements de tête...) et à l'extérieur de l'habitacle (par exemple, mouvement «anormal» des roues des automobiles ou numéro de département inscrit sur la plaque d'immatriculation n'appartenant pas à la région Ile-de-France). L'observation de l'activité de coursiers deux-roues réalisée par Van Belleghem et Bourgeois (2004) a également montré que le comportement des automobilistes était une information précieuse susceptible de fournir aux motards un diagnostic de l'exposition au risque de l'environnement. Les éléments observés portent également sur la dynamique globale du trafic environnant (espace inter-files, vitesse du trafic, espace entre deux usagers sur la même voie) et l'infrastructure (notamment la proximité d'une sortie ou entrée sur la chaussée). Les données montrent que l'espace inter-files est l'indice prioritaire extrait de l'environnement par les conducteurs observés pour circuler entre les files. Ce résultat rejoint ceux de Burge, Fox, Kouwenhoven, Rohr et Wigan (2007) qui, à partir de questionnaires, ont montré que les chaussées élargies étaient privilégiées par les motards pouvant ainsi plus facilement s'insérer entre les files. Même si cet indice peut être considéré comme l'élément principal motivant les décisions des motocyclistes, il semble que leur activité soit plus liée à la prise d'une combinaison d'éléments captés dans l'ensemble de la situation de conduite. Nos résultats soulignent ainsi la capacité des motards à savoir capter ces éléments dans l'environnement pour adapter leurs modalités de pratiques.

Les situations de remontée de files sont des situations compétitives du point de vue de la gestion de l'espace avec les autres usagers, dans des décours temporels réduits. La capacité des motards expérimentés étudiés à «balayer » la scène routière, c'est-à-dire à réaliser des changements rapides, efficaces et dans diverses directions de leur champ attentionnel, leur permet d'anticiper les éventuelles situations à risque. Les motards étudiés apparaissent ainsi comme des «experts» dans la lecture de la situation de conduite et la sélection des informations utiles. Ils développent des capacités fines en termes de discrimination perceptive. Ces compétences sur le plan de la lecture des situations, de leur compréhension et des décisions sont au cœur de la conduite en inter-files.

On retrouve d'ailleurs certaines de ces compétences chez les coursiers 2RM étudiés par Van Belleghem et Bourgeois (2004). D'après ces auteurs, les coursiers, soumis à une pratique intensive (amplitude horaire de huit heures par jour), développent des compétences comme « être capable de capter le plus grand nombre d'informations visuelles frontales et latérales » ou «savoir reconnaître des chemins à risques pour les 2RM». Même si les logiques d'activité de ces professionnels ne semblent pas exactement les mêmes que les motards non professionnels, ces compétences qui leur permettent d'anticiper les situations à risque semblent très proches. Ces déplacements professionnels méritent néanmoins pour nous des investigations spécifiques en s'appuyant sur les résultats de Van Belleghem et Bourgeois (2004) et les propositions théoriques et méthodologiques regroupées dans Hubault (2011).

En outre, ces résultats témoignent de l'implication de tous les motards étudiés dans leur conduite. On peut imaginer que cette implication est plus systématique et plus prolongée que celles des conducteurs automobiles en raison des sollicitations spécifiques de la conduite 2RM (sur les plans perceptif, cognitif et moteur). On peut d'ailleurs se poser la question du devenir des interactions automobiles/2RM dans ces situations d'inter-files (et plus généralement dans toutes les situations de conduite) avec le développement de dispositifs d'assistance et d'informations de plus en plus nombreux et sophistiqués dans les voitures. De nombreux travaux scientifiques (par exemple Hoedemaeker, \& Brookhuis, 1998 ; Nunes, \& Recarte, 2002 ; Patten, Kircher, Östlund, \& Nilsson, 2004 ; Rudin-Brown, \& Parker, 2004) 
montrent que ces dispositifs contribuent à réduire l'attention des conducteurs quant à leur environnement de conduite (et donc à la présence des motards). Un décalage encore plus important entre les niveaux d'attention de ces deux populations d'usagers est à envisager'.

\section{2.- La complexité de la législation sur les remontées de files}

L'étude qui fait l'objet de cet article est extraite d'un plus large projet commandé par la Délégation à la Sécurité et à la Circulation Routières (DSCR) du Ministère de l'Écologie, de l'Énergie, du Développement Durable et de la Mer en France. Ce projet intitulé CSC-2RM (Étude des Comportements Spontanés de Conduite des usagers de deux roues motorisés dans le trafic urbain et péri urbain) avait pour objectif de mieux connaître les comportements réels de conduite des conducteurs de motocyclettes dans l'espace parisien. La pratique de la circulation entre files étant très répandue en Ile-de-France, le projet s'est centré sur l'analyse des situations de remontées de files. Il poursuivait ainsi des visées de connaissances scientifiques (mieux connaitre la pratique de l'inter-files non encore étudiée en France en contexte naturel) et des visées transformatives (étudier la possibilité d'une législation autour des remontées de files à partir d'une analyse des pratiques réelles des motocyclistes).

Sur ce point, les résultats présentés ont montré que le différentiel de vitesse avec les automobilistes était un organisateur fort de l'activité des motards entre les files, plutôt que les allures isolées des motards. Les données déclaratives ont permis de dimensionner ces différentiels d'allures sur le boulevard périphérique parisien et de montrer les situations problématiques pour les motards. Pour répondre de manière approfondie à la demande du Législateur, ces résultats méritent néanmoins d'être complétés par (1) des mesures objectives du trafic et des vitesses des motocyclistes et (2) des analyses portant sur d'autres catégories d'usagers de $2 \mathrm{RM}$ et d'autres contextes géographiques (nos analyses ont porté exclusivement sur des motards expérimentés, utilisant des motocyclettes de grosse cylindrée, travaillant sur des plages horaires correspondant aux heures de pointe et évoluant en région parisienne).

Toutefois, les données recueillies mettent l'accent sur l'intérêt de l'étude de l'évolution de ce différentiel avec l'allure du trafic. Il s'agirait d'étudier de manière dynamique ce phénomène et de décrire cet aspect tout au long des trajets quotidiens des conducteurs. Cette piste apparait intéressante, mais très difficile à mener. En effet, l'estimation de la vitesse des files est complexe, car les situations où l'allure du trafic est constante et homogène sont rares. Le comportement et l'allure des véhicules dans chaque file semblent très "situés », c'est-à-dire qu'ils dépendent du contexte de conduite, et «dynamiques », ce contexte étant en perpétuel changement du fait des interactions entre les conducteurs. La définition des vitesses de files apparaîtra de ce fait très " grossière », " macroscopique »; elle sera forcément exprimée en termes de vitesse moyenne, une question étant de définir l'empan temporel et spatial pertinent. Il est à noter que ce point constitue une limite pour la mise en place d'une réglementation s'appuyant sur un concept de différentiel de vitesse.

\section{3.- Les implications pour l'apprentissage et la formation à la conduite}

Les résultats de cette étude permettent d'isoler un certain nombre de connaissances et de savoir-faire dont ont fait preuve les motocyclistes expérimentés dans les situations d'interfiles. Les connaissances concernent notamment les informations pertinentes à extraire de l'environnement de trafic, les associations situations/types d'informations à privilégier, les situations typiques à risque inhérentes à l'inter-files, les types de différentiels de vitesse à adopter en fonction du trafic... Les savoir-faire portent sur la capacité à rechercher et

5 Ceci est spécialement le cas pour les trajets quotidiens particulièrement congestionnés ; l'activité de conduite y est peu sollicitante, ce qui « permet » aux conducteurs d'utiliser les nouvelles technologies (téléphone, navigateur, voire télévision...). 
sélectionner les informations pertinentes de la situation de conduite et ne pas s'attarder sur les autres, la capacité de "balayer » la situation de trafic et y capter des informations situées dans la totalité de l'espace visuel, la capacité de percevoir plusieurs types d'informations simultanément, la capacité de porter son attention sur la situation de trafic tout en contrôlant son véhicule, la capacité de développer des dynamiques de couplage perception/action qui permettent d'éviter des situations potentiellement difficiles... D'après les données, il semble que l'ensemble de ces compétences fasse l'objet d'un apprentissage autonome sur le terrain. La pratique de l'inter-files n'étant pas réglementaire, elle n'est pas enseignée au cours du cursus de formation initiale en moto-école (Aupetit, 2011). Les premières expériences de conduite en inter-files et des situations critiques qui y sont associées sont donc découvertes par les motocyclistes en conditions de trafic, après l'obtention du permis. Les situations associées font l'objet d'un apprentissage «personnel», fondant l'existence d'un «curriculum caché » après la formation officielle (Perrenoud, 1995). Ceci laisse à penser que les motards expérimentés observés ont développé sur le terrain (après le permis) des compétences importantes en termes de lecture et de discrimination de l'environnement de conduite afin de percevoir les bons indices et anticiper les situations potentiellement à risque. Des compétences qu'il serait intéressant de faire passer dans la formation initiale des motards à tel point que la pratique de remontée de files est, comme on l'a vu, pratiquée par la quasi-totalité des motards en région parisienne. Ainsi guider, accompagner, soutenir et enrichir les stratégies actives d'exploration visuelle pourrait être à l'ordre du jour des enseignants d'auto-école afin de préparer les motards à la conduite réelle.

\section{5.- Conclusion}

Cette étude a permis de qualifier l'activité de motocyclistes expérimentés lors des remontées de files dans l'espace parisien. Cette pratique «tolérée » n'avait pas jusque-là fait l'objet de recherche de terrain approfondie. Les données recueillies ont montré que la circulation entre files est une pratique systématique et prolongée dans la région observée, qui représente près de $3 / 4$ du temps et de la distance parcourue lors des trajets «domicile/travail » (type de déplacement largement privilégié par les participants lors de la période d'étude). D'après les analyses, cette pratique de circulation entre files s'organise principalement autour du différentiel de vitesse avec le trafic. La situation jugée la plus risquée par les motocyclistes se présente lorsque les voitures recommencent à rouler après un arrêt momentané. Les entretiens ont également fait émerger des difficultés de cohabitation entre les usagers 2RM dans l'inter-files. Des difficultés qui s'expriment la plupart du temps en termes de confort, mais qui peuvent également altérer leur sécurité. L'autre pan de cet article a été consacré à la description de la recherche intensive d'informations à laquelle se livrent les motards. Les résultats ont montré que les focalisations des motocyclistes étaient orientées vers une pluralité d'informations hétérogènes et éparpillées dans le champ visuel du conducteur. Les motards expérimentés qui ont été observés développent d'ailleurs des compétences d'expertise dans la lecture des situations de trafic en inter-files leur permettant d'anticiper les situations potentiellement à risque.

Les développements possibles de cette étude portent notamment sur la prise en compte (1) de catégories d'usagers non (suffisamment) étudiées, (2) de conducteurs ayant des habitudes de déplacement différentes de ceux recrutés, et (3) d'autres contextes d'études.

(1) En raison des contraintes liées aux assurances pour l'autorisation de la conduite des motos instrumentées, ce sont des motocyclistes expérimentés qui ont été étudiés. Or l'analyse de motards débutants (moins de deux ans de permis moto) nous semble intéressante dans les situations de remontées de files, car les novices sont des usagers particulièrement exposés en termes d'accidentologie routière et la connaissance de leurs comportements peut contribuer à réduire ce constat. De plus, si circuler entre les files relève bien d'un apprentissage personnel alors il est intéressant dans un objectif de formation 
d'étudier les situations qui sont riches pour leur développement. Par ailleurs, l'activité des conducteurs de scooters, particulièrement présents dans l'espace parisien et donc dans les remontées de files, apparait également pertinente à investiguer. Nous pensons que les comportements des scootéristes sont pour partie différents de ceux d'un motocycliste (permis A) et donc que leur étude est essentielle, notamment dans l'objectif de fournir aux décisionnaires des éléments de connaissance sur leur activité et les problèmes qu'ils rencontrent. Enfin, les situations d'inter-files ont été étudiées dans cet article par le prisme des motocyclistes. La prise en compte du point de vue des automobilistes, à articuler avec celui des motards, semble déterminante pour disposer d'une connaissance approfondie de la situation globale d'inter-files.

(2) Les motocyclistes recrutés dans cette étude ont tous utilisé leur véhicule pour effectuer essentiellement des trajets «domicile/travail» pendant la période d'observation. Les futurs projets pourraient inclure des conducteurs avec d'autres habitudes de déplacement. Des trajets dits «de loisirs» (déplacement moins habituel que les trajets «domicile/travail » et avec des finalités différentes) ou des déplacements de travail seraient intéressants à étudier. On pense notamment aux coursiers (voir Van Belleghem et Bourgeois, 2004) et aux conducteurs de «motos taxi », dont le nombre ne cesse de grandir, qui sont soumis à des logiques d'activité différentes des motards observés dans la présente étude (exigences de prestation, rapport à l'urgence...).

(3) Les contextes d'études pourraient également être plus diversifiés. Ils pourraient être étendus en termes d'espace géographique - observer les usages dans les grandes villes de province -, et d'infrastructures - étudier les trajets en agglomération et ne pas se limiter aux autoroutes urbaines et péri-urbaines.

L'idée globale est de considérer l'ensemble des individus et des situations qui seront impactés par le changement pour alimenter les réflexions sur l'évolution de la législation qui soit satisfaisante pour tous les acteurs.

\section{REMERCIEMENTS}

Cet article est extrait du projet CSC-2RM (Étude des Comportements Spontanés de Conduite des usagers de deux roues motorisés dans le trafic urbain et péri urbain) qui a été financé par la Délégation à la Sécurité et à la Circulation Routières (DSCR) du Ministère de l'Écologie, de l'Énergie, du Développement Durable et de la Mer en France.

Les auteurs remercient Carine Pianelli et Farida Saad pour leurs contributions au projet ainsi que Jacques Riff pour la relecture des versions successives du manuscrit.

\section{BIBLIOGRAPHIE}

2BESAFE (2009/2011). Two wheeler behaviour and safety. Projet Européen (7ème PCRD). http://www.2besafe.eu

Aupetit, S. (2011). L'apprentissage de la conduite moto : analyse ergonomique et perspectives de prévention. Sarrebruck: Éditions Universitaires Européennes (ISBN 978-613-1-58535-7).

Aupetit, S., Riff, J., Buttelli, O., \& Espié, S. (2011). Former à la conduite moto. Une recherche sur les contenus d'enseignement en situation réelle. Recherche et Formation, 66, 49-64.

Aupetit, S., Riff, J., Espié, S., \& Buttelli, O. (2009). Étude de cas en situation réelle des émotions associées à la conduite moto au cours de la formation initiale. Quels enseignements pour la sécurité routière ? Recherche Transports et Sécurité, 103, 89-104.

Burge, P., Fox, J., Kouwenhoven, M., Rohr, C., \& Wigan, M. R. (2007). Modeling of motorcycle ownership and commuter usage: a UK study. Transportation Research Record: Journal of the Transportation Research Board. No. 2031. Transportation Research Board of the National Academy of Sciences, 59-68.

Cacciabue, P. C., \& Saad, F. (2008). Behavioural adaptations to driver support systems: a modelling and road safety perspective. Cognition, Technology \& Work, 10, 31-39. 
Clarke, D. D., Ward, P., Bartle, C., \& Truman, W. (2004). In-depth study of motorcycle accidents. Road Safety Research Report No. 54. Publication for Department for Transport: London.

Crundall, D., Clarke, D., Ward, P., \& Bartle, C. (2008). Car drivers' skills and attitudes to motorcycle safety: a review. Road Safety Report No. 85. Publication for Department for Transport: London.

DAMOTO (2009/2011). Détection de situations d'accidents en motocycles. Projet ANR PREDIT.

DfT (2004). Motorcycles and congestion: the effect of modal split. Phase I Report. RAND Europe.

Ducreux, B. O. (2008). Powered Two Wheelers compared with cars: driving dynamics, fuel consumption and exhaust emissions in daily use. http://www2.ademe.fr/servlet/getDoc?sort=1\&cid=96\&m=3\&id=62817\&ref=17618\&nocache=yes $\& \mathrm{p} 1=111$

Ellis, T. (2006). Inquiry into managing transport congestion. Motorcycle Riders' Association of Australia.

Glaser, B. G., \& Strauss A. L. (1967). The discovery of grounded theory. Chicago: Aldine.

Hoedemaeker, M. \& K. Brookhuis, K. (1998). Behavioural adaptation to driving with an adaptive cruise control (ACC). Transportation Research Part F, 1, 95-106.

Hubault, F. (Ed.). (2011). Activité de conduite, activité de travail. Toulouse: Octarès.

Hurt, H. H., Ouellet, J. V., \& Thom, D. R. (1981). Motorcycle accident cause factors and identification of countermeasures. Volume 1: Technical Report. Washington: Department of Transportation, National Highway Traffic Safety Administration.

Lave, J. (1988). Cognition in practice. Mind, mathematics and culture in everyday life. Cambridge: Cambridge University Press.

Leplat, J. (1990). Relations between task and activity. Element for elaborating a framework for an error analysis. Ergonomics, 33, 49-63.

Lyons, G., \& Chatterjee, K. (2008). A human perspective on the daily commute: costs, benefits and trade-offs. Transport Reviews, 28(2), 181-198.

MAIDS (2003). Motorcycle Accidents In Depth Study. ACEM (Association des Constructeurs Européens de Motocycles).

NHTSA (2000). National Adgenda for Motorcycle Safety. US Department of Transportation, National Highway Traffic Safety Administration (NHTSA) Motorcycle Safety Foundation. http://www.nhtsa.dot.gov/people/injury/pedbimot/motorcycle/00-NHT-212motorcycle/index.html.

Nunes, L. \& Recarte, M.A. (2002). Cognitive demands of hands-free-phone conversation while driving. Transportation Research Part F, 5, 133-144.

Patten, C., Kircher, A., Östlund, J., Nilsson, L. (2004) Using mobile telephones: cognitive workload and attention resource allocation. Accident Analysis and Prevention, 36(3), 341-350.

Perrenoud, P. (1995). Curriculum caché : deux paradigmes possibles. In P. Perrenoud (Ed.), Métier d'élève et sens du travail scolaire (chapitre 8) (pp.33-60). Paris: ESF.

RIDER (2005). Recherche sur les accidents impliquant un deux-roues motorisé. Projet ANR PREDIT/Fondation MAIF. Éditions: CEESAR.

Rix, G., \& Biache, M. J. (2004). Enregistrement en perspective subjective située et entretien en re-situ subjectif : une méthodologie de la constitution de l'expérience. Intellectica, 38, 363-396.

Rudin-Brown, C.M., Parker H.A. (2004). Behavioural adaptation to adaptive cruise control (ACC): implications for preventive strategies. Transportation Research Part F, 7, 59-76.

Saad, F., \& Villame, T. (1996). Assessing new driving support systems: contribution of an analysis of drivers' activity in real situations. Third Annual World Congress on Intelligent Transport Systems, Orlando, US, 14-18 October.

Sève, C., Bourbousson, J., Poizat, G., \& Saury, J. (2009). Cognition et performance collectives en sport. Intellectica, 52, 71-95.

Sperley, M., \& Pietz, A. J. (2010). Motorcycle lane-sharing. Literature review. Oregon: Department of Transportation, Research Section.

Theureau, J. (2006). Le cours d'action. Méthode développée. Toulouse: Octarès.

Theureau, J., \& Jeffroy, F. (1994). Ergonomie des situations informatisées : la conception centrée sur 
le cours d'action des utilisateurs. Toulouse: Octarès.

Van Belleghem, L., \& Bourgeois, F. (2004). Le métier de coursier et ses pratiques de prévention.

Étude ergonomique pour la prévention des risques professionnels. Rapport public. Diffusion

CRAMIF. http://www.cramif.fr/pdf/th2/prev/metier_coursier.pdf

Villame, T. (2004). Conception de systèmes d'assistance au conducteur : comment prendre en compte le caractère complexe, dynamique et situé de la conduite automobile ? Cognition située et conception de systèmes d'assistance au conducteur. Activités, 1(2), 146-169.

\begin{abstract}
RESUME
Cet article présente une analyse en contexte naturel des comportements de conduite de motocyclistes lors de leurs trajets domicile-travail en région parisienne. Elle se focalise sur la pratique des motards qui consiste à circuler entre les files de voitures, classiquement appelée «inter-files », et qui reste pour l'heure sans analyse scientifique approfondie. Dans une approche ergonomique, 11 motocyclistes ont conduit pendant un mois avec un véhicule équipé de caméras. Les enregistrements vidéo ont permis la description des contextes de conduite ainsi que la tenue d'entretiens d'auto-confrontation. Les résultats portent sur la description de leur activité de conduite, le poids de l'inter-files dans leurs déplacements quotidiens et l'analyse des éléments organisateurs de leurs comportements. Ces résultats sont discutés sur le plan de la compréhension de la pratique de l'inter-files et sur le plan de l'enrichissement des situations existantes: ils questionnent la possibilité d'une législation sur les remontées de files et montrent les intérêts de la formation à cette pratique.
\end{abstract}

\title{
MOTS-CLES
}

conduite moto, inter-files, entretien d'auto-confrontation, ergonomie, sécurité routière

\section{REFERENCEMENT}

Aupetit, S., \& Espié, S. (2012). Analyse ergonomique de l'activité de conduite moto lors de la pratique de l'inter-files en région parisienne. Activités, 9(2), 48-70, http://www.activites.org/v9n2/v9n2.pdf

Acticle soumis le 27 mars 2012, accepté pour publication le 3 juin 2012 